\title{
Multifunctional Oxide Heterostructures
}

\author{
Edited by \\ Evgeny Y. Tsymbal \\ Department of Physics and Astronomy \\ Nebraska Center for Materials and Nanoscience \\ University of Nebraska, Lincoln \\ Elbio R. A. Dagotto \\ Department of Physics and Astronomy \\ University of Tennessee, Knoxville, and \\ Materials Science and Technology Division \\ Oak Ridge National Laboratory, Oak Ridge, Tennessee \\ Chang-Beom Eom \\ Department of Materials Science and Engineering \\ University of Wisconsin, Madison \\ Ramamoorthy Ramesh \\ Department of Materials Science and Engineering \\ University of California, Berkeley
}

\section{OXFORD}




\section{Contents}

List of contributors $\quad$ xv

\section{PART I FUNDAMENTALS}

1 A brief introduction to strongly correlated electronic materials 3

E. DAGOTTO and Y. TOKURA

1.1 Motivation 3

1.2 Introduction 3

1.3 Why correlated electrons?

1.4 Control of correlated electrons in complex oxides 6

1.5 Ordering of charge, spin, and orbital degrees of freedom 11

$\begin{array}{lll}1.6 & \text { Model Hamiltonians } & 14\end{array}$

$\begin{array}{lll}1.7 & \text { Intrinsically inhomogeneous states } & 15\end{array}$

$\begin{array}{lll}1.8 & \text { Giant responses in correlated electron systems } & 18\end{array}$

1.9 Importance of quenched disorder and strain 24

1.10 Outlook for correlated-electron technology: spintronics, double
perovskites, multiferroics, orbitronics, resistance switching

$\begin{array}{ll}1.11 \text { Conclusions } & 32\end{array}$

$\begin{array}{ll}\text { Acknowledgments } & 32\end{array}$

$\begin{array}{ll}\text { References } & 32\end{array}$

2 Magnetoelectric coupling and multiferroic materials 38

Gustau CATALAN and James F. SCOTT

2.1 Introduction: magnetoelectric coupling and multiferroic materials 38

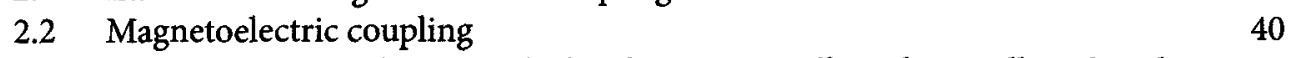

2.2.1 Linear coupling: Dzyaloshinskii-Moriya effect, electrically induced spin canting, and Shtrikman limit 41

2.2.2 Biquadratic (strain mediated) coupling $\quad 42$

2.2.3 Perovskite oxides: why are they seldom multiferroic? 43

2.3 Magnetoelectric multiferroics $\quad 44$

2.3.1 Perovskites with ferroelectricity caused by lone-pair polarization:
$\mathrm{BiFeO}_{3}$

2.3.2 Oxides with ferroelectricity caused by spins spirals: $\mathrm{TbMnO}_{3}$, $\mathrm{TbMn}_{2} \mathrm{O}_{5} \quad 46$

2.3.3 Hexagonal multiferroics: $\mathrm{YMnO}_{3} \quad 46$

2.3.4 Unconfirmed oxide multiferroics: $\mathrm{RNiO}_{3}(\mathrm{R}=$ rare earth or $\mathrm{Bi})$

$\begin{array}{lll}\text { 2.3.5 Magnetoelectric relaxors } & 48\end{array}$

2.3.6 Ferromagnetic ferroelectric fluorides $\quad 51$

2.3.7 Ferrimagnetic ferroelectrics $\quad 55$ 
Appendix 2.1 Magnetoelectric measurements

Appendix 2.2 Critical exponents in isostructural phase transitions

References

\section{PART II OXIDE FILMS AND INTERFACES: GROWTH AND CHARACTERIZATION}

3 Synthesis of epitaxial multiferroic oxide thin films

Thomas TYBELL and Chang-Beom EOM

$\begin{array}{lll}3.1 & \text { Introduction } & 73\end{array}$

$\begin{array}{lll}3.2 & \text { Substrates } & 75\end{array}$

3.2.1 Strain, orientation, and symmetry control by choice of substrate $\quad 75$

$\begin{array}{ll}\text { 3.2.2 Substrate termination and surface quality } & 77\end{array}$

$\begin{array}{lll}\text { 3.3 Strain engineering as a tool for controlling functional oxide thin films } & 79\end{array}$

3.3.1 $\mathrm{SrRuO}_{3}$ - a case study of strain engineering $\quad 80$

3.3.2 Effect of defects $\quad 86$

3.4 Vicinal control of functional properties $\quad 86$

3.4.1 $\mathrm{SrRuO}_{3}$-a case study of vicinal control of orthorhombic domain structure

3.4.2 $\mathrm{BiFeO}_{3}$-domain control of a prototype rhombohedral material by substrate miscut

3.4.3 Mono-domain samples-enabling fundamental studies and enhanced properties of $\mathrm{BiFeO}_{3}$

3.5 Conclusions

$\begin{array}{ll}\text { Acknowledgments } & 96 \\ \text { References } & 96\end{array}$

4 Synchrotron X-ray scattering studies of oxide heterostructures 99

Dillon D. FONG

$\begin{array}{llr}4.1 & \text { Introduction } & 99\end{array}$

$\begin{array}{llr}4.2 & \text { Surface X-ray diffraction } & 100\end{array}$

$\begin{array}{lll}4.3 & \text { Resonant scattering } & 105\end{array}$

$\begin{array}{lll}4.4 & \text { Anisotropic effects } & 115\end{array}$

$\begin{array}{lll}4.5 & \text { Summary } & 118\end{array}$

$\begin{array}{ll}\text { Acknowledgments } & 118\end{array}$

$\begin{array}{lr}\text { References } & 118\end{array}$

5 Scanning transmission electron microscopy of oxides 123

M. VARELA, C. LEON, J. SANTAMARIA, and S. J. PENNYCOOK

$\begin{array}{lll}5.1 & \text { Introduction to STEM } & 123\end{array}$

$\begin{array}{lll}5.2 & \text { STEM imaging } & 128\end{array}$

$\begin{array}{ll}\text { 5.2.1 Probe formation } & 129\end{array}$

$\begin{array}{ll}\text { 5.2.2 Time reversal symmetry in electron microscopy } & 130\end{array}$

$\begin{array}{ll}\text { 5.2.3 Image simulation } & 131\end{array}$

$\begin{array}{ll}\text { 5.3 Mapping materials properties through EELS fine structure } & 135\end{array}$

$\begin{array}{ll}5.4 & \text { Applications: interfaces in manganite/cuprate heterostructures } \\ \end{array}$ 
Acknowledgments $\quad 150$

References

6 Advanced modes of piezoresponse force microscopy for ferroelectric nanostructures

A. GRUVERMAN

6.1 Introduction

$\begin{array}{lll}6.2 & \text { Ferroelectric structures and size effects } & 158\end{array}$

$\begin{array}{lll}\text { 6.3 Advanced modes of PFM } & 162\end{array}$

6.3.1 Resonance-enhanced PFM: static domain imaging 162

6.3.2 Stroboscopic PFM: domain switching dynamics 165

6.3.3 PFM Spectroscopy: spatial variability of switching

$\begin{array}{llr}6.4 & \text { Summary } & 174\end{array}$

$\begin{array}{lr}\text { Acknowledgments } & 175\end{array}$

$\begin{array}{lr}\text { References } & 175\end{array}$

PART III OXIDE FILMS AND INTERFACES: FUNCTIONAL PROPERTIES

7 General considerations of the electrostatic boundary conditions in oxide heterostructures

Takuya HIGUCHI and Harold Y. HWANG

7.1 Introduction

7.2 The polar discontinuity picture

$\begin{array}{lll}7.2 .1 & \text { Stability of ionic crystal surfaces } & 185\end{array}$

$\begin{array}{ll}\text { 7.2.2 Stability of covalent surfaces } & 187\end{array}$

$\begin{array}{ll}\text { 7.2.3 Polar semiconductor interfaces } & 188\end{array}$

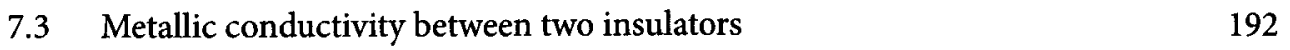

$\begin{array}{ll}\text { 7.3.1 The polar discontinuity scenario } & 193\end{array}$

7.3.2 Oxygen vacancy formation during growth 194

7.3.3 Intermixing and local bonding at the interface 194

7.3.4 Reconciling the various mechanisms 196

$\begin{array}{lll}7.4 & \text { The local charge neutrality picture } & 196\end{array}$

7.4.1 Unit-cells in ionic crystals 196

7.4.2 $\mathrm{LaAlO}_{3} / \mathrm{SrTiO}_{3}$ in the local charge neutrality picture 198

7.4.3 Coupling of polar discontinuities 199

7.4.4 Modulation doping by a proximate polar discontinuity 201

7.4.5 Advantages of the local charge neutrality picture 202

7.5 Equivalence of the two pictures 203

7.5.1 Gauss' law for infinite crystals 203

7.5.2 Gauss' law for finite crystals 204

$\begin{array}{lll}7.6 & \text { Further discussions } & 205\end{array}$

$\begin{array}{lll}7.6 .1 & \text { Effect of interdiffusion } & 205\end{array}$

$\begin{array}{ll}\text { 7.6.2 Role of correlation effects } & 207\end{array}$

$\begin{array}{ll}\text { 7.6.3 Quadrupolar discontinuity } & 207\end{array}$ 
xii Contents

$\begin{array}{lll}7.7 & \text { Summary } & 209\end{array}$

$\begin{array}{ll}\text { Acknowledgments } & 210\end{array}$

$\begin{array}{ll}\text { References } & 210\end{array}$

8 Strongly correlated heterostructures $\quad 214$

Satoshi OKAMOTO

$\begin{array}{lll}8.1 & \text { Introduction } & 214\end{array}$

$\begin{array}{lll}8.2 & \text { Theoretical description } & 218\end{array}$

$\begin{array}{lll}\text { 8.2.1 Model } & 218\end{array}$

8.2.2 Layer-extension of dynamical-mean-field theory 222

8.2.3 Auxiliary particle methods 225

8.3 Mott-insulator/band-insulator heterostructures 226

8.3.1 Lattice relaxation and charge redistribution 226

$\begin{array}{lll}\text { 8.3.2 Mott physics } & 229\end{array}$

8.4 Superlattices of under-doped-cuprate/over-doped-cuprate 232

$\begin{array}{lll}8.5 & \text { Other directions } & 236\end{array}$

8.5.1 Surface magnetism of double-exchange manganites 237

8.5.2 Transport through two-terminal strongly correlated
heterostructures

$\begin{array}{lll}8.6 & \text { Summary } & 243\end{array}$

Acknowledgments $\quad 245$

$\begin{array}{ll}\text { References } & 245\end{array}$

9 Manganite multilayers 254

Anand BHATTACHARYA, Shuai DONG, and Rong YU

$\begin{array}{lll}9.1 & \text { Motivation } & 254\end{array}$

9.2 Introduction to manganites 255

9.3 Theoretical description of manganite multilayers 256

9.4 Synthesis and structure of manganite multilayers 259

9.5 Recent progress on manganite multilayers 262

9.5.1 Phase transitions and orbital order driven by strain 262

9.5.2 Charge transfer and spin-polarized two-dimensional

9.5.3 A-site ordering in short-period superlattices $\quad 267$

9.5.4 Tuning between ferromagnetism and antiferromagnetism 273

9.5.5 Interfacial magnetism 276

9.5.6 Metal-insulator transitions 278

9.5.7 Half-manganite heterostructures: band lineup and
magnetic interactions at interfaces

$\begin{array}{lll}9.6 & \text { Conclusions and outlook } & 290\end{array}$

$\begin{array}{ll}\text { Acknowledgments } & 291\end{array}$

$\begin{array}{ll}\text { References } & 291\end{array}$

10 Thermoelectric oxides: films and heterostructures 296

Hiromichi OHTA and Kunihito KOUMOTO

$\begin{array}{lll}10.1 & \text { Introduction } & 296\end{array}$ 
10.2 p-type layered cobalt oxide: $\mathrm{Ca}_{3} \mathrm{Co}_{4} \mathrm{O}_{9}$ films 297

10.3 Heavily electron doped $\mathrm{SrTiO}_{3}$ films $\quad 300$

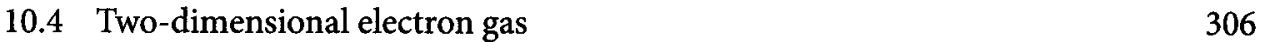

$\begin{array}{lll}10.5 & \text { Field effect thermopower modulation } & 309\end{array}$

$\begin{array}{lll}10.6 & \text { Summary } & 312\end{array}$

$\begin{array}{ll}\text { References } & 312\end{array}$

\section{PART IV APPLICATIONS}

11 High- $\kappa$ gate dielectrics for advanced CMOS 319

Suman DATTA and Darrell G. SCHLOM

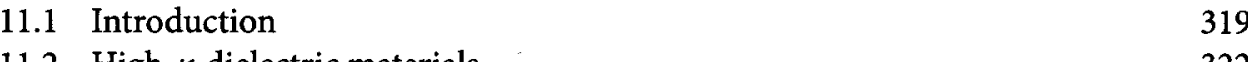

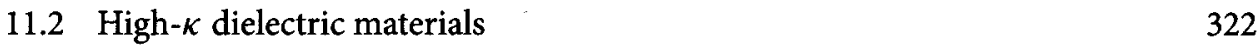

11.3 Metal-gate electrodes 323

11.3.1 Poly-depletion elimination 323

$\begin{array}{lll}\text { 11.3.2 Interfacial layer control } & 323\end{array}$

11.3.3 High- $\kappa$ phonon screening $\quad 324$

11.3.4 Metal gates with "correct" work function 325

11.4 High- $\kappa$ /metal-gate silicon FETs 326

11.4.1 Integration 326

11.4.2 Devices 329

11.4.3 Reliability 329

$\begin{array}{lll}11.5 & \text { High }-\kappa / \text { metal-gate nonsilicon FETs } & 330\end{array}$

$\begin{array}{ll}\text { 11.5.1 Integration } & 330\end{array}$

11.5.2 Devices and characterization 331

Acknowledgments $\quad 334$

References $\quad 335$

12 FeFET and ferroelectric random access memories 340

Hiroshi ISHIWARA
12.1 Overview of ferroelectric random access memories (FeRAMs)

12.2 Ferroelectric films used for FeRAMs 342

12.2.1 Properties necessary for FeRAMs 342

12.2.2 $\mathrm{Pb}(\mathrm{Zr}, \mathrm{Ti}) \mathrm{O}_{3}$ and Bi-layer structured ferroelectrics 344

12.2.3 Novel ferroelectric films with large remanent polarization $\quad 346$

12.3 Cell structure and operation principle of capacitor-type
FeRAMs

12.3.1 Cell structure of 1T1C(2T2C)-type FeRAMs 349

12.3.2 Operation principle of 1T1C(2T2C)-type FeRAMs 352

12.3.3 Other capacitor-type FeRAMs 354

12.4 Cell structure and operation principle of FET-type FeRAMs 357

12.4.1 Optimization of FeFET structure $\quad 357$

12.4.2 Data retention characteristics of FeFETs 358

$\begin{array}{lll}\text { 12.4.3 Cell array structures } & 360\end{array}$

$\begin{array}{ll}\text { References } & 362\end{array}$ 
$13 \mathrm{LaAlO}_{3} / \mathrm{SrTiO}_{3}$-based device concepts

Daniela F. BOGORIN, Patrick IRVIN, Cheng CEN, and Jeremy LEVY

13.1 Introduction

13.1.1 Semiconductor 2DEGs

13.1.2 2DEG at $\mathrm{LaAlO}_{3} / \mathrm{SrTiO}_{3}$ interface $\quad 365$

$\begin{array}{lll}\text { 13.1.3 Polar catastrophe model } & 365\end{array}$

13.1.4 Metal-insulator transition in $\mathrm{LaAlO}_{3} / \mathrm{SrTiO}_{3} \quad 366$

13.1.5 Inconsistencies with the polar catastrophe model 367

$\begin{array}{lll}13.2 & \text { Field-effect devices } & 368\end{array}$

$\begin{array}{lll}\text { 13.2.1 } & \mathrm{SrTiO}_{3} \text {-based channels } & 368\end{array}$

13.2.2 Electrical gating of $\mathrm{LaAlO}_{3} / \mathrm{SrTiO}_{3}$ structures 368

$\begin{array}{lll}\text { 13.2.3 } & \mathrm{LaAlO}_{3} / \mathrm{SrTiO}_{3} \text {-based field-effect devices } & 370\end{array}$

$\begin{array}{lll}13.3 & \text { Reconfigurable nanoscale devices } & 370\end{array}$

$\begin{array}{ll}\text { 13.3.1 Nanoscale writing and erasing } & 371\end{array}$

$\begin{array}{lll}\text { 13.3.2 "Water cycle" } & 372\end{array}$

13.3.3 $\mathrm{LaAlO}_{3} / \mathrm{SrTiO}_{3}$ as a floating-gate transistor network 373

$\begin{array}{lll}\text { 13.3.4 Quasi-0D structures } & 375\end{array}$

$\begin{array}{lll}\text { 13.3.5 Designer potential barriers } & 375\end{array}$

$\begin{array}{lll}\text { 13.3.6 SketchFET } & 376\end{array}$

13.3.7 Nanoscale photodetectors $\quad 378$

13.3.8 Integration of $\mathrm{LaAlO}_{3} / \mathrm{SrTiO}_{3}$ with silicon $\quad 379$

$\begin{array}{lll}13.4 & \text { Future prospects } & 381\end{array}$

$\begin{array}{lll}\text { 13.4.1 Room-temperature devices } & 382\end{array}$

$\begin{array}{lll}\text { 13.4.2 Information processing } & 382\end{array}$

$\begin{array}{lll}\text { 13.4.3 Spintronics } & 382\end{array}$

13.4.4 Quantum Hall regime $\quad 383$

13.4.5 Superconducting devices $\quad 383$

13.4.6 Solid-state Hubbard simulators $\quad 383$

$\begin{array}{ll}\text { References } & 384\end{array}$

$\begin{array}{ll}\text { Index } & 389\end{array}$ 